\title{
Novo registro de Doradoblatta coppenamensis Bruijning, 1959 no Brasil (Blattaria, Blattellidae) e descrição da genitália masculina.
}

\author{
Sonia Maria LOPES ${ }^{1}$; Edivar Heeren de OLIVEIRA ${ }^{1}$; Maria Carmosina de ARAÚJO²
}

\section{RESUMO}

É registrada pela primeira vez a presença de Doradoblatta coppenamensis Bruijning, 1959 nos Estados do Amazonas e Mato Grosso (Brasil); são complementados os dados originais com a descrição e ilustração da genitália masculina da espécie, e são apresentadas considerações sobre o gênero.

\section{PALAVRAS-CHAVE}

Doradoblatta coppenamensis, novo registro, Blattaria, Taxonomia.

\section{New Records of Doradoblatta coppenamensis Bruijning, 1959 from Brazil (Blattaria, Blattellidae) with Description of the male genitalia.}

\begin{abstract}
This paper records a new occurrence of Doradoblatta coppenamensis Bruijning, 1959 from Amazonas and Mato Grosso States (Brazil) and complements the original data with a description and figure of the male genitalia of the species and presents considerations about the genus.
\end{abstract}

\section{KEY WORDS}

Doradoblatta coppenamensis, new record, Blattaria, Taxonomy.

Doradoblatta é monotípico tendo sido descrito por Bruijning (1959) da região do Suriname.

A distribuição do gênero, até então restrita àquela região, neste trabalho é ampliada para o Brasil, nas regiões norte e centro-oeste, respectivamente nos Estados do Amazonas e Mato Grosso. Com a identificação de alguns exemplares depositados na coleção do Museu Nacional e de um exemplar do Instituto Nacional de Pesquisas da Amazônia como Doradoblatta coppenamensis Bruijning, 1959, foi possível complementar a descrição original com a caracterização e ilustração da genitália masculina da espécie.

A localidade de Parque das Laranjeiras no Amazonas onde um dos espécimes foi coletado era uma região de floresta de terra firme, isolada por uma floresta secundária (Ready et al., 1985). Atualmente está toda fragmentada e transformada em um bairro residencial de Manaus. A coleta foi feita manualmente em palmeiras.

A cidade de Sinop, MT, local de coleta dos outros exemplares, localiza-se a 500 quilômetros ao norte de
Cuiabá, às margens da BR-163, a Cuiabá-Santarém. O relevo é plano, suavemente ondulado, com raros pontos de erosão, favorável ao desenvolvimento da agricultura. A vegetação é de floresta tropical, do tipo ombrófila (site da Prefeitura Municipal de Sinop).

Para a observação das peças genitais foram utilizadas técnicas para dissecção, descritas em Lopes \& Oliveira (2000). A designação das peças genitais e a classificação taxonômica seguiram Mckittrick (1964). Nas descrições foram utilizadas abreviaturas para os locais de depósito do material estudado: MNRJ (Museu Nacional do Rio de Janeiro) e INPA (Instituto Nacional de Pesquisas da Amazônia).

\section{Catálogo de gênero e espécie de Doradoblatta Bruijning, 1959}

Doradoblatta Bruijning

Doradoblatta Bruijning, 1959: 86-87, figs. 28-34

Espécie-tipo: D. coppenamensis Bruijning, 1959

\footnotetext{
${ }^{1}$ Departamento de Entomologia, Museu Nacional, UFRJ, Quinta da Boa Vista, São Cristóvão, RJ, Brasil, CEP 20940-040, sonialf@acd.ufrj.br ${ }^{2}$ Instituto Nacional de Pesquisas da Amazônia, Manaus, AM, Brasil CEP 69011-970, carmo@inpa.gov.br
} 


\section{ACTA AMAZONICA}

NOVO REGISTRO DE Doradob/atta coppenamensis BRUIJNING, 1959

NO BRASIL (BLATTARIA, BLATTELLIDAE) E DESCRIÇÃO DA GENITÁLIA MASCULINA.

\section{Doradoblatta coppenamensis Bruijning}

D. coppenamensis Bruijning, 1959: 86-87, figs. 28-34

Holótipo: macho (Leiden Museum) Suriname

Registros Geográficos: Suriname, Brasil (AM, MT).

Coloração amarelo-esbranquiçada com três faixas transversais castanho-escuras, sendo uma na base, uma na região mediana e outra no ápice da tégmina (Fig. 9). A cabeça e o pronoto são como representados nas figuras 1 e 2 . Unhas tarsais simples; pulvilos somente no $4^{\circ}$ artículo. Abdome do macho com a superfície dorsal modificada, fortemente esclerotinizada; a placa supra-anal simétrica, curta, transversa, com parapróctos bem desenvolvidos e cercos longos e ciliados (Fig. 3); placa subgenital de ápice arredondado e ciliado, com estilos simétricos, curtos, largos e ciliados, inseridos em uma reentrância na região mediana no ápice da placa, com uma projeção arredondada da mesma entre eles (Figs. 4 e 5).

Genitália $\left(\sigma^{\top}\right)$ - Falômero esquerdo com dois braços simétricos em forma de $\mathrm{V}$ invertido; estrutura mediana esclerotinizada e bastante evidenciada (Fig. 6). Esclerito
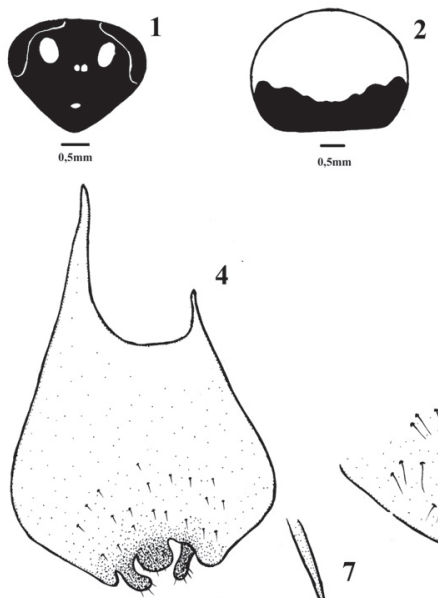
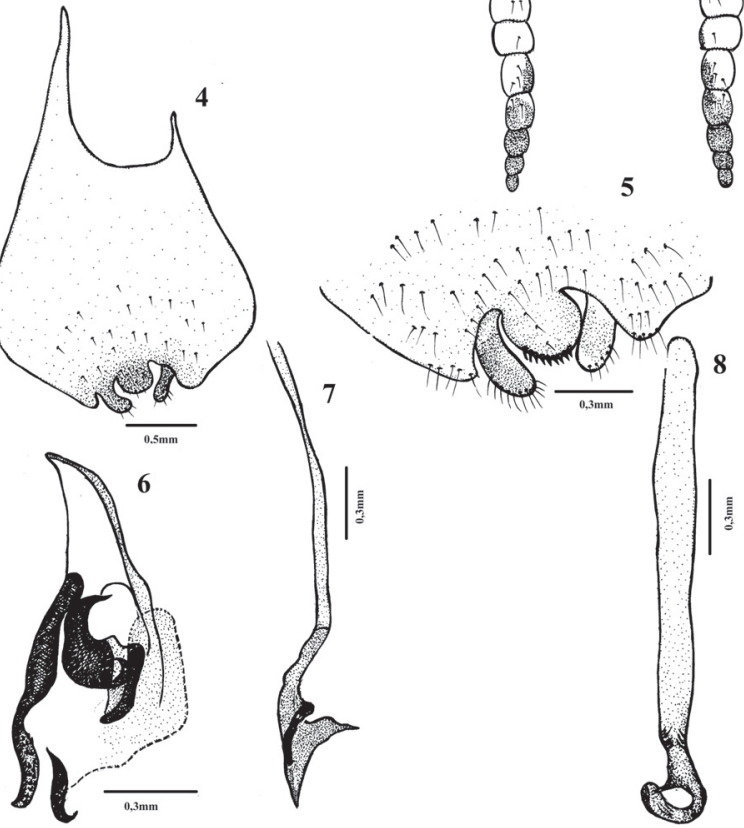

Figuras 1 a 8 - Doradoblatta coppenamensis Bruijning, 1959 ( ). 1. Cabeça. vista ventral; 2 , pronoto, vista dorsal; 3 , placa supra-anal, vista dorsal; 4, placa subgenital, vista ventral; 5 , detalhe dos estilos, vista ventral; 6 , falômetro esquerdo; 7 , esclerito mediano; e ápice do esclerito mediano, vista ventral; 8 , falômetro direito, vista ventral. mediano apicalmente espatulado e fortemente esclerotinizado (Fig. 7). Falômero direito em forma de gancho apicalmente bem definido e muito esclerotinizado (Fig. 8).

Material examinado: BRASIL, Amazonas, Manaus, Parque das Laranjeiras, 18/VI/1981, Jorge Arias col. (macho) (INPA); Mato Grosso, Sinop, 12³1'S e 55'37'W, $350 \mathrm{mts}$ alt., IX/1974, Alvarenga \& Roppa cols. (2 machos) (MNRJ).

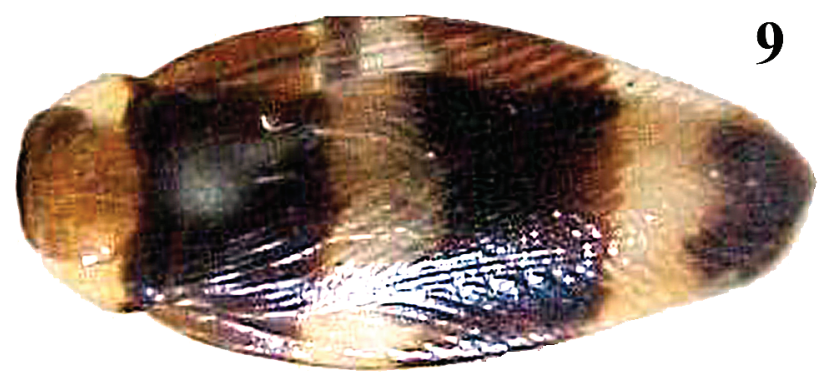

Figura 9 - Habitus: Doradoblatta coppernamensis Bruijning, 1959.

\section{AGRADECIMENTOS}

À Dra. Janira Martins Costa, professora do Museu Nacional, pelo apoio técnico. Ao Dr. José Albertino Rafael, pesquisador do INPA, pelo empréstimo do material para estudo.

\section{BIBLIOGRAFIA CITADA}

Bruijning, C. F. A. 1959. The Blattidae of Surinam. Studies on the Fauna of Suriname and other Guyanas, 2 (4): 1-103.

Lopes, S.M; Oliveira, E. H. 2000. Espécie Nova de Eublaberus Hebard, 1919 do Estado de Goiás, Brasil e notas sobre $E$. marajoara Rocha e Silva-Albuquerque, 1972 (Blaberidae, Blaberinae). Boletim do Museu Nacional, Nova Série, Zoologia, 433: 1-5.

Mckittrick. F.A. 1964. Evolutionary studies of cockroaches. Cornell University Agricultural Experiment Station Memoir, 389: 1-197.

Prefeitura Municipal de Sinop 2002. Site acessado em 31/07/2003: bttp://www.sinop.mt.gov.br.

Ready, P. D.; Arias, J. R.; Freitas, R. A. 1985. A pilot study to control Lutzomya umbratilis (Diptera: Psychodidae), the major vetor of Leishmania braziliensis guyanensis, in a peri-urban rainforest of Manaus, Amazonas State, Brazil. Memórias do Instituto Oswaldo Cruz, 80(1): 27-36.

RECEBIDO EM 13/03/2003

ACEITO EM 18/02/2004 